\title{
Identification of the Elastic Properties of Isotropic and Orthotropic Thin-Plate Materials with the Pulsed Ultrasonic Polar Scan
}

\author{
M. Kersemans • A. Martens • N. Lammens • \\ K. Van Den Abeele • J. Degrieck • F. Zastavnik • \\ L. Pyl • H. Sol • W. Van Paepegem
}

Received: 20 November 2013 / Accepted: 4 February 2014 / Published online: 1 March 2014

(C) Society for Experimental Mechanics 2014

\begin{abstract}
Already in the early 1980's, it has been conjectured that the pulsed ultrasonic polar scan (P-UPS) provides a unique fingerprint of the underlying mechanical elasticity tensor at the insonified material spot. Until now, that premise has not been thoroughly investigated, nor validated, despite the opportunities this would create for NDT and materials science in general. In this paper, we report on the first-ever implementation of an inverse modeling technique on the basis of a genetic optimization scheme in order to extract quantitative information from a P-UPS. We validate the optimization approach for synthetic data, and apply it to experimentally obtained polar scans for annealed aluminum, cold rolled DC-06 steel as well as for carbon fiber reinforced plastics. The investigated samples are plate-like and do not require specific preparation. The inverted material characteristics show good agreement with literature, micro-mechanical models as well as with results obtained through conventional testing procedures.
\end{abstract}

Keywords Ultrasound · Polar scan · Metals and composites · Elastic properties $\cdot$ Characterization

M. Kersemans $(\varangle) \cdot$ N. Lammens $\cdot$ J. Degrieck $\cdot$ W. Van Paepegem Department of Materials Science and Engineering, Ghent University, Technologiepark-Zwijnaarde 903, 9052 Zwijnaarde, Belgium e-mail: mathias.kersemans@ugent.be

A. Martens $\cdot$ K. Van Den Abeele

Department of Physics, Catholic University of Leuven, Campus

Kortrijk - KULAK, Etienne Sabbelaan 52, 8500 Kortrijk, Belgium

F. Zastavnik $\cdot$ L. Pyl $\cdot$ H. Sol

Department Mechanics of Materials and Construction, Vrije

Universiteit Brussel, Pleinlaan 2, 1050 Brussels, Belgium

\section{Introduction}

The high strength-to-weight ratio, in combination with a tunable stiffness, make composite materials very attractive for a range of high-tech applications, which include wind turbine blades, primary components in aerospace industry, etc. For monitoring and inspecting these critical structures, a variety of approaches are available [1-5], among which the most widespread concerns the application of ultrasound. Besides the classical ultrasonic $\mathrm{C}$-scan, which has already proven its usefulness in detecting defects, delaminations as well as material heterogeneities, several more advanced techniques can quantitatively monitor the mechanical performance in order to ensure the designed functionality of a composite structure. At present, (nonlinear) resonant ultrasonic spectroscopy ((N)RUS) is a well-established technique to infer elasticity constants [6], and even monitor damage progression [7-9], of anisotropic materials. However, (N)RUS is a resonance based technique, in which the specifically prepared sample needs to be mounted in a device, making it useless for in situ determination of the material characteristics. The latter restriction also holds for techniques which are based on time measurements of bulk waves because measurements have to be taken at specific material orientations [10-15]. Besides, for anisotropic media the propagation- and energy velocity are decoupled, making the correct recording and subsequent interpretation of experimental data a rather difficult task. Plane wave transmission characteristics have been used by various research teams to extract the (visco)elastic material properties $[16,17]$. Basically, the sample is insonified at a few well-chosen oblique incidence angles with a broadband ultrasonic pulse which resembles a plane wave [18]. The transmitted wave field is recorded in time, normalized to the reference wave field, and further analyzed in the frequency domain. In this way the transmission coefficient is obtained as a function of 
the frequency. The resulting transmission coefficient has sharp minima which relate to the condition for efficient stimulation of Lamb waves, while the global level of the transmission coefficient is a measure for the attenuation. Another closely related technique which gained a lot of popularity over the last years concerns the application of guided waves, such as Lamb waves, Rayleigh waves, etcetera, which probe the structure under investigation. The stimulated guided wave interrogates the structure upon propagation, hence the recorded signal contains a watermark of the material characteristics [19-21]. The main disadvantage of such a guided wave based approach concerns the complexity of the data acquisition and the extensive post-processing in order to obtain the envisioned results, especially when the investigated structure is for example notflat/curved [22-24]. Besides, the guided wave technique provides a global representation of the material characteristics, while it is often needed to obtain the material characteristics at a local material spot.

Already in the early 80's, the ultrasonic polar scan (UPS) technique was introduced [25] as a promising tool for NDT and material research. UPS's are created by insonifying a material spot for a 2D range of oblique incidence angles, and simply gathering the transmitted (or if necessary the reflected) maximum pulse amplitude [25-27]. A schematic of the method is shown in Fig. 1(a). By mapping the recorded amplitudes in polar representation $(\varphi, \theta)$, a polar scan image is formed in which intriguing contours emerge (see Fig. 1(b)). Each point in a UPS image corresponds to a unique incidence angle $\psi(\phi, \theta)$, while the assigned color pigment is a measure for the transmitted maximum amplitude. The characteristic contours in a UPS image are linked to the generation of critically refracted (quasi-)bulk waves in the case of sound pulses [28], and thus are from a physics point of view directly connected to the mechanical properties of the sample material at the targeted spot. In the remainder of this study, we denote a pulsed ultrasonic polar scan by P-UPS. More recently, it has been shown that the operational regime of the P-UPS can be expanded to the recording of the associated time-of-flight (TOF) value [29, 30], to the recording of backscattered waves [31] as well as to the analysis of the associated phase in case of a harmonic version of the polar scan (H-UPS) [27], in order to gain supplementary information of the insonification spot. In this paper however, we limit ourselves to amplitude recorded P-UPS results in a transmission setup. Since a single material spot is interrogated in an omnidirectional way, no prior knowledge about the material is required to obtain the envisioned data. The very simple data-acquisition in which only the maximum transmission pulse amplitude needs to be stored as well as the straightforward post-processing, makes it a very appealing and simple technique to extract material characteristics. Especially when considering the enormous amount of redundant data in a P-UPS experiment, from which an inversion procedure would obviously benefit to efficiently extract material properties with a high degree of accuracy. Finally, it is worth noting that numerical computations indicate that the P-UPS technique works equally well in a reflection setup [28].

It has been already conjectured in several studies that the characteristic contours in a P-UPS image are a unique encryption of the local elastic material properties $[25,26,28,29$, 32-35]. Nevertheless this statement has not yet been comprehensively investigated, nor verified, which immediately defines the scope of the present paper. We investigate numerically as well as experimentally the possibility to extract the local material characteristics from an amplitude recorded PUPS.

The next section gives a short description of the implemented system identification procedure, which is based on an optimization procedure by means of a genetic algorithm (GA). Inversion results are first presented and discussed for synthetic data, in order to explore the opportunities as well as the limitations of the method for material characterization.
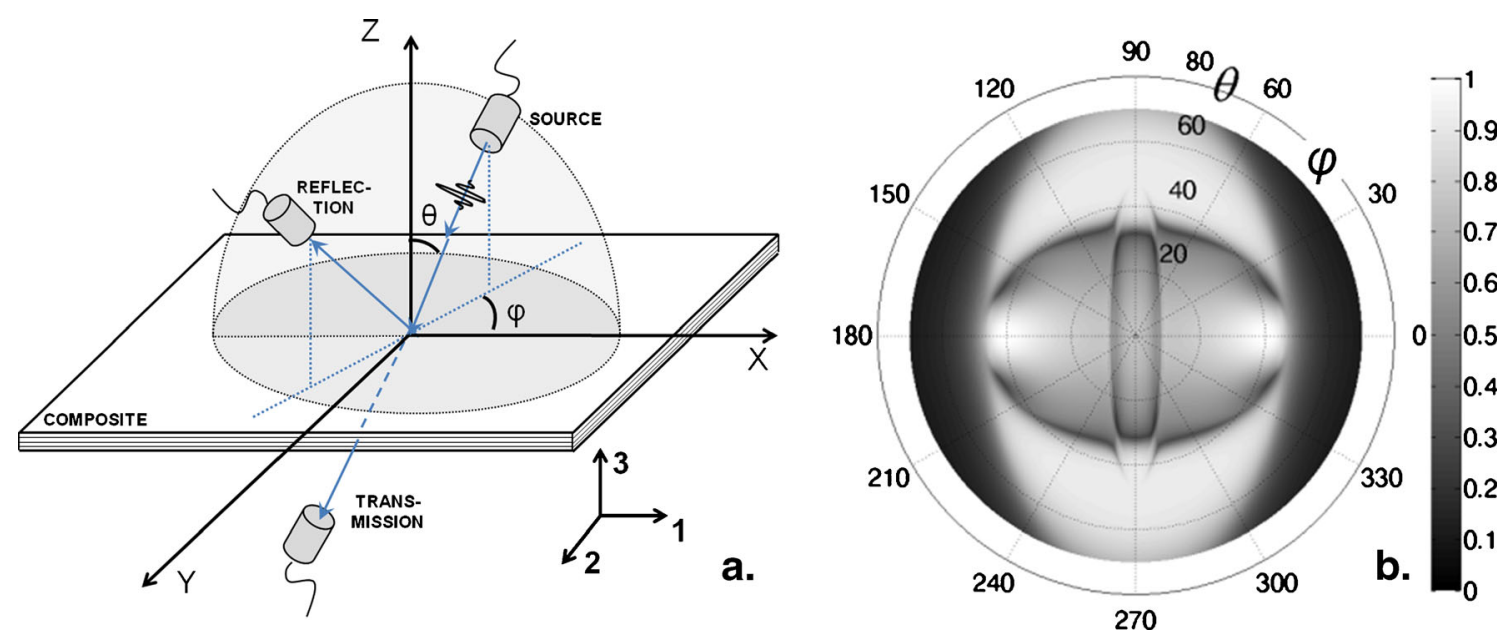

Fig. 1 Schematic of the UPS principle (a), numerically computed P-UPS for a $[0]_{8}$ carbon/epoxy laminate (b) 
Finally, the system identification procedure is applied to experimentally obtained P-UPS experiments for several annealed aluminum samples in different mechanical health, cold-rolled DC-06 steel samples and an autoclave manufactured $[0]_{8}$ carbon fiber reinforced plastic plate.

\section{Optimization Procedure}

Since the characteristic contours in a P-UPS image are a representation of the in-plane critical bulk wave angles for all polarization states [28], it is clear that the inversion procedure should be based on the well-known Christoffel equation. Indeed, the characteristics of plane wave propagation in an infinite elastic anisotropic solid are effectively described by the Christoffel equation (Einstein summation convention) [36].

$\left(\rho \omega^{2} \delta_{i m}-C_{i k l m} k_{k} k_{l}\right) u_{m}=0$

with $\rho$ the density, $\omega$ the circular frequency, $\delta$ the Kronecker delta, $\boldsymbol{C}$ the elasticity tensor, $\boldsymbol{k}$ the unit wave vector and $\boldsymbol{u}$ the polarization of the mechanical wave. This equation leads to an orthogonal classical eigenvalue problem for which three independent solutions exist in case of a symmetry higher than or equal to monoclinic symmetry. The eigenvalue problem is solved by demanding nontrivial solutions, which is equivalent to setting the determinant of the coefficient matrix equal to zero:

$\left|\rho \omega^{2} \delta_{i m}-C_{i k l m} k_{k} k_{l}\right|=0$

With this, the computed eigenvalues can be entered back into the system of original homogeneous equations to calculate the eigenvectors and confirm the wave type and character, i.e. pure or quasi. Application of Snell-Descartes' law for externally borne sound finally leads to the determination of the critical bulk wave angles which are then compared with the characteristic contours in a P-UPS image. By selecting an appropriate set of elastic constants, the computed in-plane bulk wave angle profiles can then be matched with the PUPS contours.

In general, composite materials are anisotropic of nature, in which orthotropy can be considered the most common symmetry class. A full description of the orthotropic nature can be given by knowledge of nine elastic constants. Hence it is clear that an appropriate set of these nine constants, to obtain a good fit with the contours hidden in the P-UPS image, cannot be simply obtained by trial and error. This is especially true considering that the elastic constants describe a highly nonlinear space. In this paper, an optimization scheme is employed which relies on the principle of Darwin, i.e. an abstraction is made in terms of a population in which the hierarchy is determined by the survival of the fittest (see
Fig. 2). A set of different $C_{i j}$ 's corresponds to a complete population in the genetic algorithm (GA), while a single elasticity tensor $C_{i j}$ corresponds to an individual in the population. The choice for a genetic optimization procedure is mainly triggered by its great flexibility for optimizing nonlinear multi-dimensional problems, its capability to converge to the near optimum of the problem regardless the initial guess and the fact that a GA can be easily parallelized, reducing the computational effort. An even more important advantage of GA concerns its capability to not remain trapped in a suboptimal solution since a population covers different parts of the parameter space. GA's have already been successfully employed in various studies for inverting (synthetic) wave velocity data in order to obtain a measure of the mechanical properties [12, 14, 20, 37].

Basically we start with a random initial population, consisting of 100 individuals, i.e. 100 unique elasticity tensors $C_{i j}$, from which the corresponding critical bulk wave profiles are computed through application of Christoffel's equation in combination with the Snell-Descartes law. The parameter space needs bounds in which the GA has a complete freedom of selectivity in order to obtain the optimized set of elasticity constants $C_{i j}$. Of course, these bounds should be chosen as narrow as possible to speed up the inversion process, while assuring that the space contains the optimal solution. Typically, we set the bounds at $\pm 90 \%$ of the estimated elasticity constants $C_{i j}$ to make sure that the parameter space contains the optimal solution. The increment at which the elasticity parameters can be updated, is chosen fairly small in order to improve the accuracy. The computed critical bulk wave angle profiles are then simply superposed on the P-UPS from which a measure of their fitness is obtained by summing the interpolated P-UPS amplitudes at the exact coordinates of the computed curves, and this for all polarization states

$\sum_{k} \sum_{l} A_{I}\left(\phi_{k}, \theta_{k, \text { Christ }}^{l}\right)$

with $A_{I}\left(\phi_{k}, \theta_{k}^{l}\right)$ the interpolated transmitted pulse amplitude at coordinates $\left(\phi_{k}, \theta_{k, \text { Christ }}^{l}\right)$ in which $\phi_{k}$, respectively $\theta_{k, \text { Christ }}^{l}$, represents the polar direction, respectively the computed critical bulk wave angle for a given elasticity tensor $C_{i j}$ and $l=$ $Q L, Q S H, Q S V$ denotes the quasi-longitudinal, the quasi-shear horizontal and the quasi-shear vertical polarization state. This is illustrated in Fig. 3 for an arbitrary elasticity tensor $C_{i j}$.

Since the computed profiles should merge to the lowamplitude contours in the P-UPS image in order to obtain a qualified elasticity tensor $C_{i j}$, it is clear that the above criterion has to be minimized:

$\min \left(\sum_{k} \sum_{l=Q L, Q S H, Q S V} A_{I}\left(\phi_{k}, \theta_{k, \text { Christ }}^{l}\right)\right)$ 
Fig. 2 Schematic of the optimization algorithm

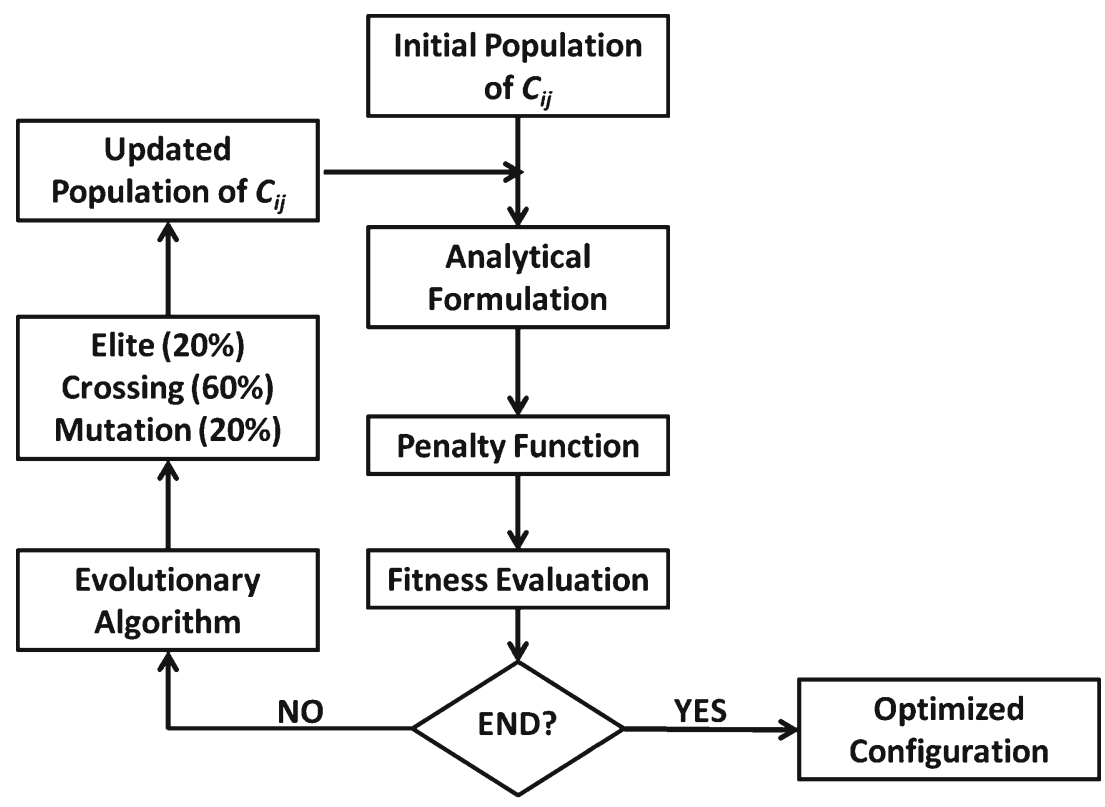

The fact that the superimposed lines do not fully match the P-UPS contours in Fig. 3 clearly reveals that the current elasticity tensor $C_{i j}$ cannot be regarded as the optimal elasticity tensor $C_{i j}$.

It is noted that the P-UPS contours are also characterized by a high local curvature value, hence the fitness criterion can also be adapted in order to maximize the summed curvature values. In fact, this criterion is also applied to the P-UPS data of the metallic samples because of the accelerated convergence.

The above procedure is executed for each elasticity tensor $C_{i j}$ in the current population, the obtained fitness values for

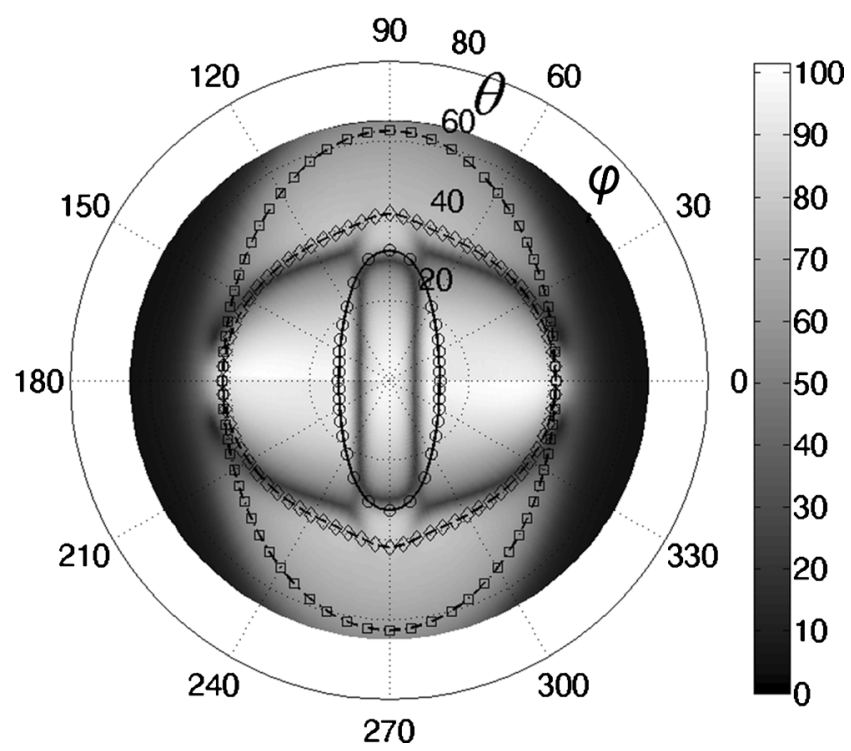

Fig. 3 P-UPS experiment for a $[0]_{8}$ carbon/epoxy laminate. The critical bulk wave angle profiles, computed for an arbitrary elasticity tensor $C_{i j}$, are superposed that population are then sorted in descending order. After this, the current population generates the next population by producing descendants. This step is basically dictated by three descendant-generation rules. First, those individuals having superior fitness values are directly recruited in the next generation according to the principle of elitism, thus forming the elite children $(20 \%)$. Second, crossover children are formed by randomly mixing the characteristics of any two individuals (60\%). Lastly, the next population is further complemented with random mutations of an individual by randomly flipping bit values from 0 to 1 and vice versa, thus resulting in a set of mutated individuals $(20 \%)$. Once the next generation is created, the fitting procedure is repeated in order to obtain a fitness value of the updated generation. This loop is repeated for many updated populations, until convergence is obtained. The stopping procedure is activated when two criterions are satisfied at the same time. The variation of the fitness value of both the current best individual and the mean of the population should be sufficiently small over ten subsequent generations. Once these conditions are fulfilled, the procedure is stopped and the optimized elasticity tensor $C_{i j}$ is obtained.

Nevertheless, our first inversion results revealed that our basic assumption, being the fact that the P-UPS contours provide a one-to-one relationship with the in-plane critical bulk wave angles [28], is not exactly valid. This is numerically demonstrated in Fig. 4 for a hypothetical transversal isotropic material. The P-UPS simulation is performed according to the recursive stiffness matrix method [27, 38, 39]. The spectral frequency content of a typical ultrasonic broadband pulse is accounted for by means of a Fourier integral. It can be seen that the superposed critical bulk wave angle profiles slightly deviate from the characteristic contours in the numerically computed P-UPS, although both have been computed with 
Fig. 4 Simulated P-UPS at $f d=$ $5 \mathrm{MHz} \times 1 \mathrm{~mm}$ for a hypothetical orthotropic material with the computed critical bulk wave angle profiles superposed for the range $\phi \in\left[0^{\circ}, 180^{\circ}\right]$ (a) and magnification to accentuate the discrepancy between the position of the P-UPS contours and the critical bulk wave angle profiles (b)

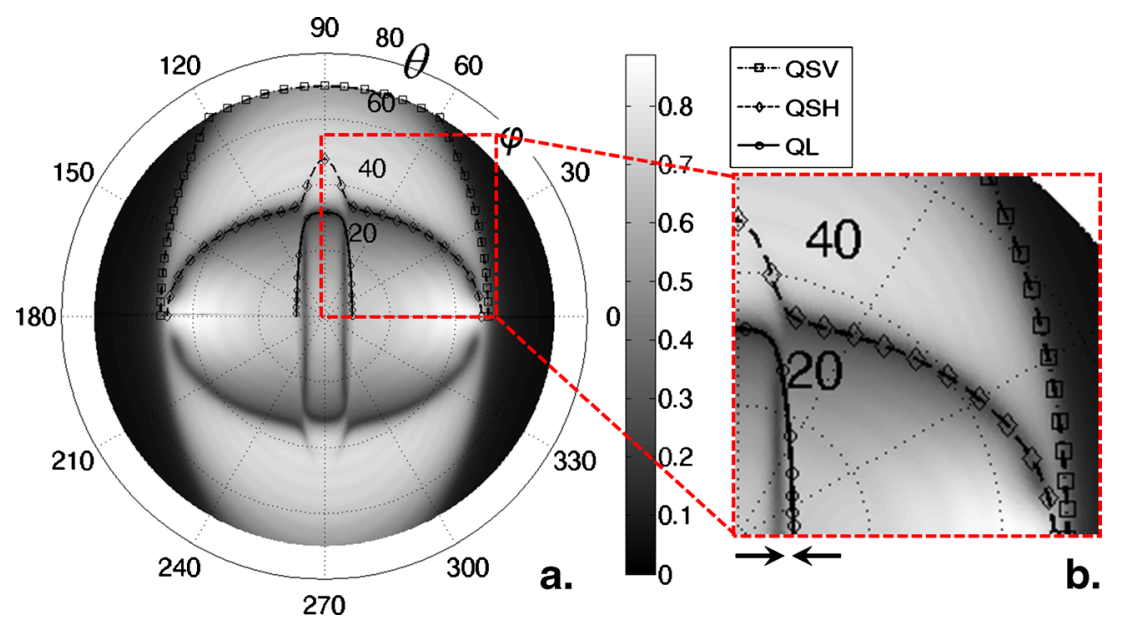

the same set of material parameters (listed in Table 1). Especially for the inner contour, which relates to the stimulation of the in-plane propagating quasi-longitudinally (QL) polarized bulk wave, and in extension to the Young's modulus $E_{i i}$, this discrepancy can be clearly observed along $\varphi=0^{\circ}$ (see Fig. 4(b)). The position of this P-UPS minimum is rather dictated by that incident angle $\theta$ where the shear horizontal wave component in the solid becomes dominant over the longitudinally polarized component [40]. Although the angular deviation can be considered to be small, it is critical to account for in order to obtain a correct and accurate inversion of the elasticity tensor $C_{i j}$ from a P-UPS image. Especially when considering that the here considered composite material has a very high mechanical stiffness along $\varphi=0^{\circ}$, which implies that the inner contour has a small incident angle $\theta$. According to Snell-Descartes' law, any small change in incident angle then results in a substantial change in wave speed and in extension to the underlying mechanical stiffness. Therefore, the system identification procedure on the basis of the Christoffel solutions needs an extension to account for this discrepancy.

The extension of the inversion procedure basically relies on the same scheme as described in Fig. 2. Though, an initial population is defined based on the elasticity tensor $C_{i j}$ obtained in the first inversion procedure on the basis of the Christoffel solutions. From this set of elasticity tensors $C_{i j}$, P-UPS sectors at a few fixed polar angles $\varphi$ are computed. The P-UPS simulations are performed according to the recursive stiffness matrix method $[27,38,39]$. By allowing small perturbations in the elasticity tensor $C_{i j}$, the local minima in the computed P-UPS sections are matched with the observed minima in the P-UPS experiment. The main reason why only a few sectors are considered, instead of the full P-UPS image, is mainly triggered by computation constraints. Indeed, the

Table 1 Inversion results for synthetic data: mean value \pm standard deviation (relative error \%). The last row is put in gray as it has been obtained through symmetry considerations

\begin{tabular}{|c|c|c|c|c|c|}
\hline & \multirow{2}{*}{$\begin{array}{c}\text { Input } \\
{[G P a]}\end{array}$} & \multicolumn{4}{|c|}{ Output [GPa] } \\
\hline & & Scatter $0 \%$ & Scatter $5 \%$ & Scatter $15 \%$ & Scatter $30 \%$ \\
\hline$C_{11}$ & 117.43 & $\begin{array}{c}117.46 \pm 0.50 \\
(+0.03 \%)\end{array}$ & $\begin{array}{c}118.05 \pm 3.05 \\
(+0.53 \%)\end{array}$ & $\begin{array}{c}114.99 \pm 5.91 \\
(-2.12 \%)\end{array}$ & $\begin{array}{c}130.55 \pm 23.93 \\
(+10.05 \%)\end{array}$ \\
\hline$C_{12}=C_{13}$ & 4.99 & $\begin{array}{c}5.03 \pm 0.07 \\
(+0.96 \%)\end{array}$ & $\begin{array}{c}5.05 \pm 0.38 \\
(+1.20 \%)\end{array}$ & $\begin{array}{c}4.96 \pm 0.70 \\
(-0.54 \%)\end{array}$ & $\begin{array}{l}5.76 \pm 2.39 \\
(+13.39 \%)\end{array}$ \\
\hline$C_{22}=C_{33}$ & 13.94 & $\begin{array}{c}13.96 \pm 0.08 \\
(+0.15 \%)\end{array}$ & $\begin{array}{c}14.01 \pm 0.10 \\
(+0.50 \%)\end{array}$ & $\begin{array}{c}13.97 \pm 0.30 \\
(+0.19 \%)\end{array}$ & $\begin{array}{c}14.76 \pm 1.32 \\
(+5.57 \%)\end{array}$ \\
\hline$C_{44}$ & 3.90 & $\begin{array}{c}3.91 \pm 0.07 \\
(+0.27 \%)\end{array}$ & $\begin{array}{c}3.89 \pm 0.06 \\
(-0.28 \%)\end{array}$ & $\begin{array}{c}3.92 \pm 0.11 \\
(+0.48 \%)\end{array}$ & $\begin{array}{c}3.98 \pm 0.29 \\
(+1.95 \%)\end{array}$ \\
\hline$C_{55}=C_{66}$ & 7.19 & $\begin{array}{c}7.16 \pm 0.00 \\
(-0.44 \%)\end{array}$ & $\begin{array}{c}7.13 \pm 0.04 \\
(-0.87 \%)\end{array}$ & $\begin{array}{c}7.06 \pm 0.13 \\
(-1.82 \%)\end{array}$ & $\begin{array}{c}6.62 \pm 0.93 \\
(-8.56 \%)\end{array}$ \\
\hline$C_{23}=C_{22}-2 C_{44}$ & 6.14 & $\begin{array}{c}6.14 \pm 0.21 \\
(0.00 \%)\end{array}$ & $\begin{array}{c}6.23 \pm 0.08 \\
(-1.47 \%)\end{array}$ & $\begin{array}{c}6.13 \pm 0.47 \\
(-0.16 \%)\end{array}$ & $\begin{array}{l}6.8 \pm 0.89 \\
(-10.75 \%)\end{array}$ \\
\hline
\end{tabular}


simulation of a P-UPS, having the same angular resolution as a P-UPS experiment, involves the computation of more than 1 million incidence angles [41]. Moreover, since an ultrasonic pulse is employed, it is critical to account for its temporal frequency content to obtain a realistic simulation. The latter is taken care of by means of a Fourier integral. To speed up this process, the integral is replaced by its discrete counterpart being the fast Fourier transform (FFT), in which typically around 400 dominating frequencies are selected in the spectrum of the ultrasonic pulse. Finally, the simulated P-UPS has to be fitted to the recorded P-UPS. Although the first optimization step on the basis of Christoffel's equation provides a good estimation of the elasticity tensor $C_{i j}$, still numerous PUPS simulations with updated material properties are needed in order to fine-tune the material properties. With current high performance numerical facilities, such a full P-UPS inversion would take several days. On the contrary, by starting from the elasticity tensor $C_{i j}$ obtained in the first optimization step and considering only a few sectors of the P-UPS in the second optimization step, we could limit the processing time of the complete inversion procedure to less than $5 \mathrm{~min}$ on a standard computer.

In order to demonstrate the described procedure to extract the local in-plane mechanical parameters from a P-UPS, results are first presented for synthetic data. A numerically computed P-UPS is generated for a transversal isotropic material with stiffness parameters listed in Table 1 . The imaginary elasticity parameters (which describes attenuation characteristics) have been fixed at $2.5 \%$ of the real part of the elasticity tensor. The inversion procedure is then applied to the P-UPS simulation in order to retrieve the employed mechanical properties. The parameter bounds for the optimization procedure are fixed at $\pm 90 \%$ of the actual material properties. Because the initial population of the GA is composed of a set of randomly chosen individuals and the optimization procedure does not flow in a deterministic way, it is clear that the inversion procedure is a stochastic process in which the determined optimum cannot be considered to be fixed. To assure the appropriateness of the implemented inversion procedure for the problem considered here, the inversion has been applied ten times to the numerically computed P-UPS image from which the standard error $\sigma$ is determined. In addition, the inversion procedure has been applied to synthetic data with added scatter. A scatter level of $5 \%, 15 \%$ and $30 \%$ has been applied to the transmission amplitudes. Figure 5(a-b) displays the numerically computed P-UPS, used as an input for the inversion procedure, with no scatter and scatter level $30 \%$.

The optimization results for the synthetic data are grouped and listed in Table 1. Note that the last row $\left(C_{23}\right)$ has been indirectly obtained by applying the well known symmetry relation of transversal isotropic media. As such, it provides an extra measure of the appropriateness of the here considered inversion procedure. It is clear that the optimized constants for the non-scattered data are very close to the input constants, while the standard error $\sigma$ is fairly small. Although the error and standard deviation of the inversion results for the data with scatter increase, it can be readily verified that good inversion results are obtained up to $15 \%$ scatter, indicating the robustness of the method. Considering the large amount of useful data in a P-UPS, this could have been foreseen. Further increase of the scatter level up to $30 \%$ deteriorates the accuracy of the inversion results significantly. However, $30 \%$ scatter can be considered to be extremely high in most realistic environments. Hence, we extracted the elastic parameters $C_{11}, C_{12}, C_{22}, C_{44}$ and $C_{55}$ in a stable and robust way by inverting the (scattered) P-UPS data of the hypothetical material. For a material with a lower symmetry class than transversal isotropy, one can further decouple $C_{66}$ from $C_{55}$. However, to obtain the remaining orthotropic parameters $\left(C_{13}, C_{23}\right.$ and $\left.C_{33}\right)$ a second P-UPS has to be performed in another material symmetry plane. Nevertheless, since most structural components are plate-like, these characteristics are of minor importance.

\section{Experimental Results}

In this section, the inversion procedure is applied to amplitude recorded P-UPS experiments. The experiments have been obtained by means of an in-house developed robot having five axes of freedom. The axes are driven by brushless DC motors which have been programmed with LABVIEW in order to insonify a single material spot at multiple incidence angles in a fully automatic way. High precision incremental position encoders have been installed. Besides accurate position feedback, they also trigger the ultrasonic circuit and the data-acquisition module. Typically, more than 1 million unique incidence angles $\psi(\phi, \theta)$ are involved in a P-UPS experiment, resulting in an angular step size of $0.05^{\circ}$ for the incident angle $\theta$. Two identical unfocused shock-wave immersion transducers (General Electric), with diameter $d=13 \mathrm{~mm}$, are used in order to generate and subsequently record ultrasonic waves. The piezoelectric crystal generates a broadband ultrasonic pulse, having a central frequency $f_{c}=5 \mathrm{MHz}$. The distance between both transducers is more or less $D=130 \mathrm{~mm}$, the sample under investigation is placed in between them. The limited diameter of the transducers invokes that the investigated materials have to be sufficiently thin because of the characteristic beam shift upon transmission. The application of an acoustic mirror to neutralize the beam shift $[39,40]$ is not considered because this introduces several other problems [41]. Better would be to use transducers with a larger diameter, or to perform the experiment in reflection. With the current setup and transducers, the accuracy of the experiment can only be guaranteed for samples with a thickness below $2 \mathrm{~mm}$. The inversion procedure is applied to different extruded and 


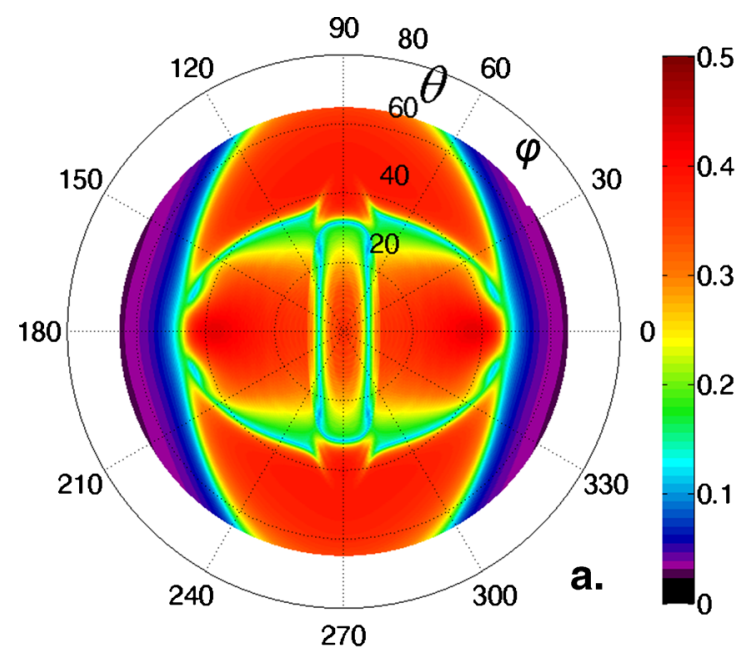

Fig. 5 P-UPS simulation: no scatter (a) and $30 \%$ scatter (b)

annealed aluminum samples, cold-rolled DC-06 steel samples and a carbon fiber reinforced laminate possessing a large degree of anisotropy. For the inversion of experimentally obtained P-UPS images, the bounds are fixed at $\pm 90 \%$ of the estimated material properties.

\section{Annealed Aluminum}

Two annealed extruded aluminum samples with different chemical composition, having a respective thickness $d_{1}=$ $1.5 \mathrm{~mm}\left(\mathrm{Al}_{1}\right)$ and a thickness $d_{2}=1.4 \mathrm{~mm}\left(\mathrm{Al}_{2}\right)$, are scanned. The density of the samples was measured according to ASTM D792 (2008): "Standard Test Methods for Density and Specific Gravity (Relative Density) of Plastics by Displacement", a value of $\rho=2676.6 \mathrm{~kg} / \mathrm{m}^{3}$ for $\mathrm{Al}_{1}$ and $\rho=2693.2 \mathrm{~kg} / \mathrm{m}^{3}$ for $\mathrm{Al}_{2}$ is determined. The recorded P-UPS for $\mathrm{Al}_{2}$ is shown in Fig. 6. Obviously, the recorded P-UPS of $\mathrm{Al}_{1}$ is very similar and is therefore omitted. The mechanical isotropy of the aluminum sample $\mathrm{Al}_{2}$ is clearly reflected in the circular shape of the characteristic patterns. Furthermore, only two characteristic contours are discerned because of the degeneration of the QSH and QSV wave in case of isotropy.

Because of human error when mounting the sample in the experimental setup, the recorded experiment has to be centered first to the polar axes [42]. First the geometrical midpoint $\psi\left(\phi_{0}, \theta_{0}\right)$ of the characteristic contours is determined

$$
\left(\phi_{0}, \theta_{0}\right)={ }^{1} /_{P} \sum_{l=1}^{P}\left(\tan ^{-1}\left(\frac{\sum_{k=1}^{N} \theta_{k, M I N}^{l} \sin \phi_{k}}{\sum_{k=1}^{N} \theta_{k, M I N}^{l} \cos \phi_{k}}\right), \frac{\sqrt{\sum_{k=1}^{N} \theta_{k, M I N}^{l^{2}}}}{N}\right)
$$

with $\mathrm{P}$ the number of different polarization states (QL, QSH and QSV), $\mathrm{N}$ the number of experimental data along

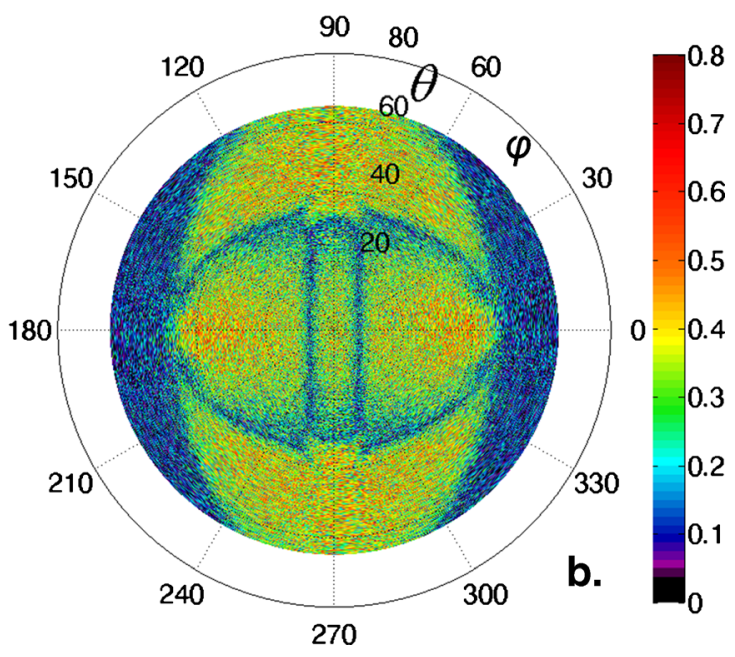

$\varphi$-direction and $\theta_{k, M I N}^{l}$ the determined position of the characteristic contours along polar direction $\phi_{k}$.

Subsequently, a translation of the experimental data in polar coordinates $\psi(\phi, \theta) \rightarrow \psi^{*}\left(\phi+\phi_{0}, \theta+\theta_{0}\right)$ is performed

$$
\begin{aligned}
\theta^{*} & =\sqrt{\theta^{2}+\theta_{0}^{2}-2 \theta \theta_{0} \cos \left(\phi-\phi_{0}\right)} \\
\phi^{*} & =\tan ^{-1}\left(\frac{\theta \sin \phi-\theta_{0} \sin \phi_{0}}{\theta \cos \phi-\theta_{0} \cos \phi_{0}}\right)
\end{aligned}
$$

The inversion procedure is then applied ten times to the centered P-UPS experiment in order to extract the local material parameters. Because of the isotropy of the aluminum sample, it is clear that the inversion procedure can be limited to the optimization of two material parameters, being $C_{11}$ and $C_{44}$. Nevertheless, we expand the parameter space for the

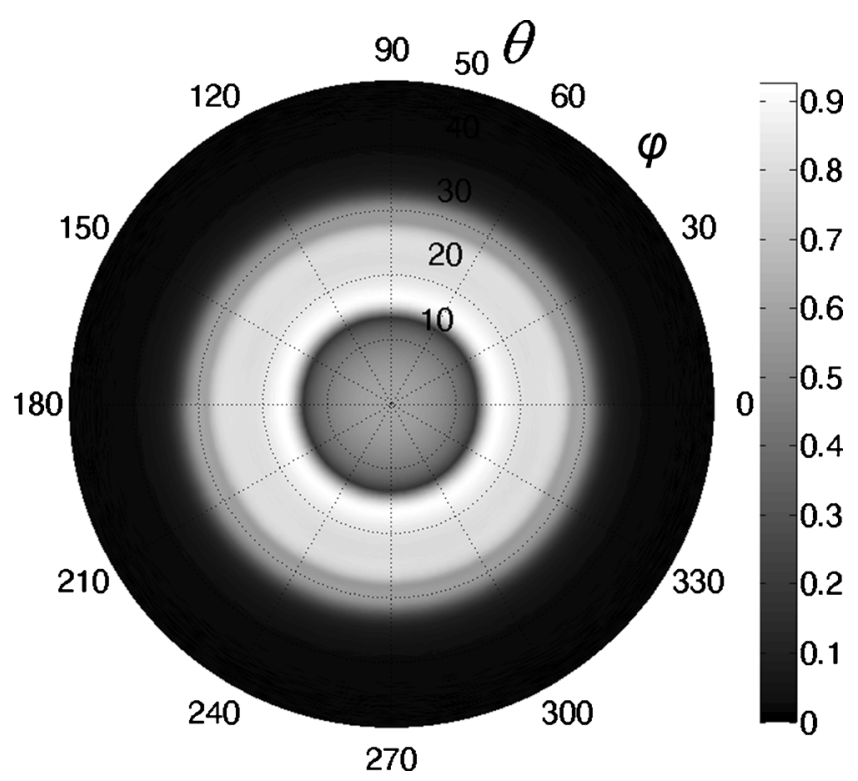

Fig. 6 Recorded P-UPS at $f d=5 \mathrm{MHz} \times 1.4 \mathrm{~mm}$ for the $\mathrm{Al}_{2}$ sample 
inversion procedure to $C_{11}, C_{12}, C_{22}, C_{44}, C_{55}$ and $C_{66}$, in order to confirm the mechanical symmetry of the aluminum samples. The results are listed in Table 2. The number between parentheses represents the standard deviation of the inversion procedure. Since we obtained slightly deviating material parameters for both aluminum samples, each sample has been scanned a second time at a different location. It is clear that the second P-UPS scans yield very consistent inversion results in comparison with the results obtained from the first P-UPS scans. Since both aluminum samples are from a different class of alloy, it can be stated that the observed deviation in material parameters between both samples $\mathrm{Al}_{1}$ and $\mathrm{Al}_{2}$ is a reflection of their slightly different chemical composition, rather than experimental or numerical error. The consistency between $C_{11}$ and $C_{22}$ on the one hand, and between $C_{44}, C_{55}$ and $C_{66}$ on the other hand clearly confirms the isotropic nature of both the aluminum samples $\mathrm{Al}_{1}$ and $\mathrm{Al}_{2}$. This was expected because both samples have been extruded, followed by a recrystallizing annealing process. To further ascertain the isotropic nature of the aluminum samples, one can evaluate the Zener anisotropy ratio $Z=\frac{2 C_{44}}{C_{11}-C_{12}}$, in which $Z=1$ corresponds to isotropy. For $\mathrm{Al}_{1}$, respectively $\mathrm{Al}_{2}$, we obtain a Zener anisotropy ratio of $Z=1.00065$, respectively $Z=1.00368$, hence confirming the isotropic nature of the investigated aluminum samples. For isotropic symmetry, the inverted $C_{i j}$ parameters can be easily converted in order to obtain the engineering constants $E$ and $\nu$. Table 3 lists the extracted engineering constants for the different aluminum samples. The error (in percent) with generally accepted engineering constants for aluminum, being $E=70 \mathrm{Gpa}$ and $\nu=0.34$, is added. It is clear that the P-UPS inversion results show excellent agreement with literature. Nevertheless, the statement that the slight differences in elastic properties are introduced by a different chemical composition could not be verified independently because of the limited sensitivity of a standard tensile test setup (in the order of $\pm 2 \mathrm{GPa}$ ).

A further analysis is performed to the influence of a plastic deformation on the elastic material parameters of aluminum
Table 3 Extracted engineering constants for the different aluminum samples. The error $\varepsilon$ has been determined with respect to the literature values $E=70 \mathrm{GPa}, \nu=0.34$

\begin{tabular}{llllll}
\hline & & $E[\mathrm{GPa}]$ & $\varepsilon_{E}[\%]$ & $\nu[-]$ & $\varepsilon_{\nu}[\%]$ \\
\hline Literature & & $69-71$ & - & $0.33-0.35$ & - \\
$\mathrm{Al}_{1}$ & P-UPS 1 & 68.41 & $-2.27 \%$ & 0.3397 & $-0.09 \%$ \\
& P-UPS 2 & 68.37 & $-2.33 \%$ & 0.3393 & $-0.21 \%$ \\
$\mathrm{Al}_{2}$ & P-UPS 1 & 70.62 & $+0.89 \%$ & 0.3400 & $+0.00 \%$ \\
& P-UPS 2 & 69.86 & $-0.20 \%$ & 0.3427 & $+0.79 \%$ \\
$\mathrm{Al}_{2}$ & P-UPS 1 & 70.24 & $+0.34 \%$ & 0.3412 & $+0.35 \%$ \\
Plastic strain 1 $_{2}$ & P-UPS 2 & 70.25 & $+0.36 \%$ & 0.3412 & $+0.35 \%$ \\
$\mathrm{Al}_{2}$ & P-UPS 1 & 70.24 & $+0.34 \%$ & 0.3413 & $+0.38 \%$ \\
Plastic strain 2 & P-UPS 2 & 69.85 & $-0.21 \%$ & 0.3426 & $+0.76 \%$ \\
& & & & & \\
\hline
\end{tabular}

sample $\mathrm{Al}_{2}$. Therefore, aluminum sample $\mathrm{Al}_{2}$ has been loaded in a tensile machine into its plastic regime. After unloading and demounting the sample from the tensile machine, the sample has been scanned twice (near the region where plastic shear bands were observed visually) under similar circumstances as was done for the virgin sample. These steps were repeated for a second and higher tensile load level, inducing an even larger plastic deformation of the aluminum sample. The extracted material parameters for the plastically loaded aluminum samples have been added to Table 3. Compared to the results of the virgin aluminum sample, it can be stated that plastic straining does not affect the elastic material parameters. It is known from classical mechanics that a modest plastic deformation introduces strain hardening, but does not influence the elastic material properties.

Hence, the current results clearly demonstrate both the accuracy and reproducibility of the P-UPS technique for identifying the material parameters of isotropic media, even after a plastic loading cycle. The minor deviations between experiments and literature can be primarily attributed to five causes: (i) the presence of inevitable experimental errors

Table 2 Inversion results for the different aluminum samples. The inversion procedure has been applied ten times, from which the mean value and standard deviation (between brackets) is obtained

\begin{tabular}{llllllll}
\hline & & $C_{11}[\mathrm{GPa}]$ & $C_{12}[\mathrm{GPa}]$ & $C_{22}[\mathrm{GPa}]$ & $C_{44}[\mathrm{GPa}]$ & $C_{55}[\mathrm{GPa}]$ & $C_{66}[\mathrm{GPa}]$ \\
\hline $\mathrm{Al}_{1}$ & P-UPS 1 & $105.1093(0.275)$ & $54.1320(0.255)$ & $105.2437(0.5037)$ & $25.5220(0.000)$ & $25.5220(0.000)$ & $25.5220(0.000)$ \\
& P-UPS 2 & $104.9295(0.000)$ & $53.8854(0.000)$ & $104.9292(0.001)$ & $25.5220(0.000)$ & $25.5220(0.000)$ & $25.5220(0.000)$ \\
$\mathrm{Al}_{2}$ & P-UPS 1 & $108.6877(0.000)$ & $55.9814(0.000)$ & $108.6878(0.000)$ & $26.3532(0.000)$ & $26.3532(0.000)$ & $26.3532(0.000)$ \\
& P-UPS 2 & $108.6877(0.000)$ & $56.6576(0.000)$ & $108.6877(0.000)$ & $26.2076(0.000)$ & $26.0092(0.000)$ & $26.0151(0.000)$ \\
$\mathrm{Al}_{2}$ & P-UPS 1 & $108.6857(0.005)$ & $56.3431(0.029)$ & $108.7333(0.063)$ & $26.1832(0.000)$ & $26.1832(0.000)$ & $26.1832(0.000)$ \\
Plastic strain 1 & P-UPS 2 & $108.6838(0.009)$ & $56.3877(0.120)$ & $108.8246(0.2481)$ & $26.1832(0.000)$ & $26.1832(0.000)$ & $26.1832(0.000)$ \\
$\mathrm{Al}_{2}$ & P-UPS 1 & $108.6878(0.000)$ & $56.3213(0.000)$ & $108.6877(0.000)$ & $26.1832(0.000)$ & $26.1832(0.000)$ & $26.1832(0.000)$ \\
Plastic strain 2 & P-UPS 2 & $108.6410(0.002)$ & $56.6085(0.002)$ & $108.6087(0.004)$ & $26.1481(0.000)$ & $25.9250(0.000)$ & $26.0990(0.000)$ \\
& & & & & & &
\end{tabular}


and varying laboratory conditions, (ii) the fixed angular resolution $\left(\Delta \theta=0.05^{\circ}\right)$ of the P-UPS recording, (iii) errors induced by the inversion procedure, (iv) the variation in chemical composition of different aluminum samples and (v) the variation of the elastic material properties within a single aluminum sample. The next section extends the analysis to the inversion of experimentally recorded P-UPS of cold-rolled steel samples.

\section{Cold-Rolled DC-06 Steel}

Cold-rolled DC-06 steel, with a measured density of $\rho=7704.7 \mathrm{~kg} / \mathrm{m}^{3}$ (ASTM D792), is a very mild steel which is very popular in automobile industry because of its excellent formability. It is known for its pronounced anisotropic plastic behavior, which is best reflected in the famous cup drawing test. Most materials form four cusps, while the DC-06 steel forms six cusps because of its anisotropic plastic properties [43]. It is very tempting to generalize this plastic anisotropy to the elastic regime. Though, such a generalization would definitely yield incorrect results. Here, the P-UPS technique is employed to check whether the cold-rolled DC-06 steel possesses any anisotropy in the elastic region.

The recorded P-UPS for the DC-06 steel is shown in Fig. 7. At first sight it could be perceived that the characteristic patterns are circles, thus reflecting isotropic symmetry. However, close observation reveals that the contours are slightly pressed inwards at $\varphi=90+\mathrm{n} \pi$, thus having an elliptical shape. In the light of previous results, this implies that a certain mechanical anisotropy is present in the DC-06 steel. Similar to the aluminum samples, an inversion is performed to the $C_{11}, C_{12}, C_{22}, C_{44}, C_{55}$ and $C_{66}$ parameters. The results are

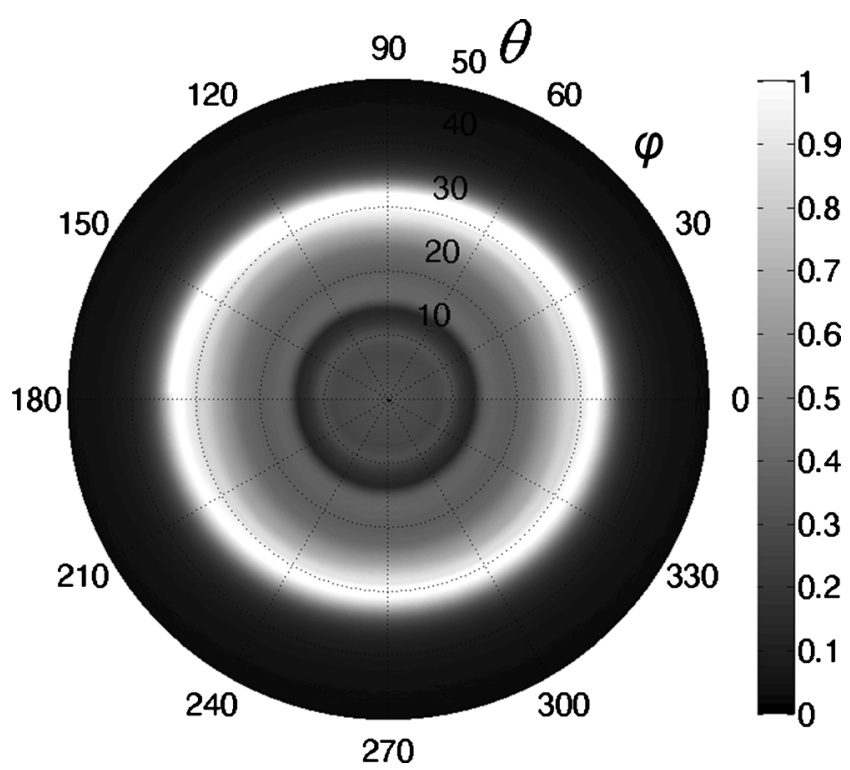

Fig. 7 Recorded P-UPS at $f d=5 \mathrm{MHz} \times 0.8 \mathrm{~mm}$ for DC-06 steel listed in Table 4. Evaluation of the inverted results reveals some interesting particularities. The small deviation between $C_{11}$ and $C_{22}$ indicates that the DC-06 samples cannot be considered to be strictly isotropic. This is further confirmed by the discrepancy between $C_{44}, C_{55}$ and $C_{66}$. Unfortunately, no representative elastic data for the cold-rolled DC-06 steel can be found in literature, which makes the validation of the inverted material parameters difficult. Anyway, our inversion results are qualitatively supported:

- Multiple P-UPS experiments at different DC-06 samples have been performed to obtain a standard deviation of the inverted $C_{i j}$ parameters. It can be readily verified that the inverted parameters systematically show a low standard deviation, which indicates the consistency of the inverted values.

- A finite element model, on the basis of the viscoplastic selfconsistent code (VPSC) [44], has been setup in order to estimate the elastic properties of material after applying a similar rolling process. Since the chemical composition as well as the prehistory of the DC-06 steel is unknown, we assumed a bcc crystal structure with 10,000 random textured grains. Hence the initial stiffness tensor on the poly-crystal level is isotropic. After rolling, the stiffness tensor on the poly-crystal level changed towards a slightly orthotropic symmetry in agreement with the P-UPS inverted values.

- Finally, few papers already demonstrated the orthorhombic nature of rolled steel at the poly-crystal level [45]. Though, the level of orthorhombic symmetry can be considered to be very subtle.

On the other hand, when computing the Zener anisotropy ratio when considering averaged $C_{i j}$ parameters, a value of $Z^{*}=\frac{2\left(C_{44}+C_{55}+C_{66}\right) / 3}{\left(C_{11}+C_{22}\right) / 2-C_{12}}=1.00732$ is obtained indicating that the material possesses a symmetry close to isotropy. Then, by simplifying the slightly orthotropic nature of the steel sample to isotropy, the following engineering constants are obtained: $E=189.68 \pm 0.59 \mathrm{GPa}$ and $\nu=0.345 \pm 0.001$. As the Poisson ratio $\nu$ is higher than could be expected for steel, we performed additional tensile tests on the DC-06 steel in order to complement the ultrasonically determined values. A stiffness of $E=192.36 \pm 1.64 \mathrm{GPa}$ and a Poisson ratio of

Table 4 Inversion results for the DC-06 steel samples. Several DC-06 samples have been scanned, from which the mean value and standard deviation is obtained

\begin{tabular}{lllllll}
\hline & $\begin{array}{l}C_{11} \\
{[\mathrm{GPa}]}\end{array}$ & $\begin{array}{l}C_{12} \\
{[\mathrm{GPa}]}\end{array}$ & $\begin{array}{l}C_{22} \\
{[\mathrm{GPa}]}\end{array}$ & $\begin{array}{l}C_{44} \\
{[\mathrm{GPa}]}\end{array}$ & $\begin{array}{l}C_{55} \\
{[\mathrm{GPa}]}\end{array}$ & $\begin{array}{l}C_{66} \\
{[\mathrm{GPa}]}\end{array}$ \\
\hline DC-06 & 301.7904 & 157.0102 & 294.2795 & 71.6643 & 69.8521 & 71.5696 \\
& \pm 2.2681 & \pm 1.7143 & \pm 1.8371 & \pm 0.2084 & \pm 0.3714 & \pm 0.5127 \\
& & & & & &
\end{tabular}


Table 5 Material properties for the carbon/epoxy laminate. First column contains the data provided by the supplier (CIBA-GEIGY), the second column the parameters provided by Soden [38], the third column contains parameters obtained within our research team, complemented with estimated values, the fourth and fifth column represents micro-mechanical homogenization results [40-42], and the last column lists the P-UPS inverted material parameters complemented with parameters obtained from symmetry relations. Bold values have been obtained in our lab in a direct way, italic values are obtained indirectly, while (italic) values are estimations

\begin{tabular}{lllllll}
\hline $920-\mathrm{CX}$ & Ciba-Geigy & $\begin{array}{l}\text { Soden } \\
{[38]}\end{array}$ & $\begin{array}{l}\text { Degrieck } \\
{[39]}\end{array}$ & $\begin{array}{l}\text { Chamis } \\
{[40]}\end{array}$ & $\begin{array}{l}\text { Puck [41] } \\
\text { Foye [42] }\end{array}$ & P-UPS \\
\hline$V_{f}[\%]$ & 55 & 60 & $\mathbf{5 5}$ & 55 & 55 & 55 \\
$E_{11}[\mathrm{GPa}]$ & 115.1 & 138 & $\mathbf{1 1 9 . 1 3}$ & 128.74 & 128.74 & $\mathbf{1 1 8 . 4 2}$ \\
$E_{22}[\mathrm{GPa}]$ & - & 11 & $\mathbf{8 . 8 5}$ & 10.50 & 12.23 & $\mathbf{1 0 . 1 8}$ \\
$E_{33}[\mathrm{GPa}]$ & - & 11 & 8.85 & 10.50 & 12.23 & 10.18 \\
$\nu_{12}[-]$ & - & 0.28 & $\mathbf{0 . 3 0 6}$ & 0.319 & 0.319 & $\mathbf{0 . 3 2 8}$ \\
$\nu_{13}[-]$ & - & 0.28 & 0.306 & 0.319 & 0.319 & 0.328 \\
$\nu_{23}[-]$ & - & 0.40 & $(0.475)$ & 0.367 & 0.428 & 0.473 \\
$G_{12}[\mathrm{GPa}]$ & - & 5.5 & $(5.50)$ & 4.51 & 4.89 & $\mathbf{6 . 2 5}$ \\
$G_{13}[\mathrm{GPa}]$ & - & 5.5 & $(5.50)$ & 4.51 & 4.89 & $\mathbf{5 . 8 6}$ \\
$G_{23}[\mathrm{GPa}]$ & - & 3.93 & $(3.00)$ & 3.84 & 4.28 & $\mathbf{3 . 3 9 8}$ \\
\hline
\end{tabular}

$\nu=0.349 \pm 0.004$ have been determined, which are in excellent agreement with the P-UPS values. Though, it is clear that caution is required with these 'isotropic values'. The P-UPS reveals that the investigated DC-06 steel samples possesses a small degree of elastic orthotropy. Nevertheless, for most engineering applications it can be considered as being effectively isotropic in the elastic regime.

\section{Orthotropic Carbon/Epoxy Laminate}

In this section, the experimental analysis is extended to the inversion of the local elasticity constants of a transversal isotropic carbon fiber reinforced plastic. The investigated $\left[0^{\circ}\right]_{8}$ carbon/epoxy laminates are composed of unidirectional prepreg layers, with thickness $d \approx 0.14 \mathrm{~mm}$ and a measured fiber volume fraction $V_{f} \approx 55 \%$, from the type Fibredux 920 CX (company Ciba-Geigy). The laminates have been autoclave manufactured according to the instructions of the supplier, i.e. a $60 \mathrm{~min}$ cycle at a temperature of $T=125^{\circ} \mathrm{C}$ and a pressure of $p=3.5 \mathrm{bar}$. The density of the sample is measured according to ASTM D792, a value of $\rho=1528.6 \mathrm{~kg} / \mathrm{m}^{3}$ is determined. The elastic material properties of the material are listed in Table 5. The values in bold are measured values, while the values in italic have been obtained indirectly. The mechanical properties provided by the supplier are listed in the first column of Table 5. The second column lists the parameters for a similar unidirectional carbon/epoxy laminate though for a fiber volume fraction of $V_{f}=60 \%$ [46]. The third column contains values obtained within our research team by means of conventional testing procedures [47]. The fourth, respectively fifth column represent the results of the analytical micro-mechanical homogenization model of Chamis [48], respectively of Puck-Foye [49, 50], in which the fiber properties [51] and epoxy properties (www.Hexcel.com: data sheet HexPly $920125^{\circ} \mathrm{C}$ curing epoxy matrix) are used as input. It is clear that the different sets of parameters are not really consistent to each other, and therefore cannot be regarded as the actual material properties, but rather provide a magnitude.

The P-UPS has been recorded at $f d=5 \mathrm{MHz} \times 1.1 \mathrm{~mm}$ at an arbitrary material spot and is shown in Fig. 8(a). The sample has been placed randomly in the test setup, hence the fiber direction obviously deviates from the $0^{\circ}$-direction of the PUPS setup. Hence, in addition to the inversion procedure described for isotropic solids, here we first extract the fiber direction of the laminate by searching for the orientation angle $\varphi_{\text {fiber }}$ at which the inner characteristic contour in the P-UPS recording has a minimum incident angle $\theta$. Indeed, a minimal incident angle $\theta$ of the inner contour corresponds to a maximal
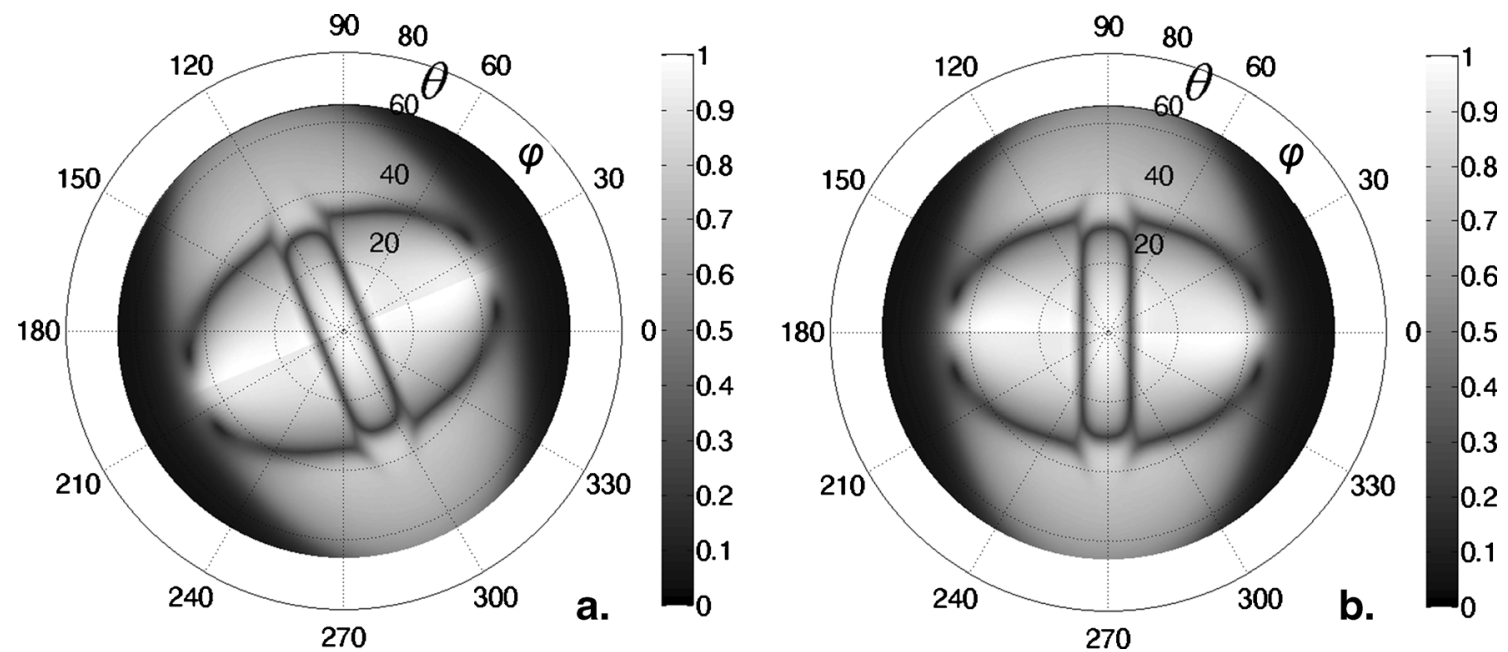

Fig. 8 P-UPS recorded at $f d=5 \mathrm{MHz} \times 1.1 \mathrm{~mm}$ for an autoclave manufactured $\left[0^{\circ}\right]_{8}$ carbon/epoxy laminate (a) and after rotation over $\varphi_{\text {fiber }}(\mathbf{b})$ 
sound speed for the in-plane propagating QL polarized wave, which implies a high elasticity modulus in that particular direction. The latter obviously arises from the specific orientation of the designed carbon fiber reinforcement. The P-UPS experiment is then rotated over the extracted fiber orientation angle $\varphi_{\text {fiber }}$ (see Fig. 8(b)). In this way, the optimization procedure is significantly accelerated since the principal axes of orthotropy are known and consequently can be fixed at $\varphi=$ $0^{\circ}$ and $\varphi=90^{\circ}$. Such a characterization of the local fiber direction per se is actually very interesting for the composite manufacturing industry where the stacking process of laminae is often done manually. The quality of the stacking procedure directly influences the stiffness properties, hence a spot-tospot inspection of the local fiber direction is an easy way to assure the designed functionality of the composite structure. Even more, local wrinkling of reinforced fibers, which is an important failing mechanism for composite structures, can also be easily detected and evaluated.

In theory, the manufactured $\left[0^{\circ}\right]_{8}$ carbon/epoxy laminate belongs to the symmetry class of transversal isotropy, and thus is described by five independent elasticity parameters. However, a real composite seldom exhibits pure symmetry because of (i) human error in the layer stacking procedure, (ii) small perturbations in the pressure and/or temperature distribution of the autoclave manufacturing cycle, (iii) production faults in the supplied prepreg material and (iv) unequal spatial distribution of the fibers in 2- and 3-direction due to the applied pressure level during manufacturing. Hence, it can be expected that the shear properties in the 12- and 13-direction are not equivalent. Therefore, we decoupled both and performed the system identification procedure to the optimization of $C_{11}, C_{12}, C_{22}, C_{44}, C_{55}$ and $C_{66}$. The P-UPS obtained material properties are listed in the last column of Table 5. Compared to the information of the supplier as well as to the conventionally determined material constants, satisfying agreement is found. Comparison with the micro-mechanically predicted material properties reveals that the optimized set of material parameters are in the same range. Nevertheless, it is clear that a quantitative comparison cannot be made. The P-UPS extracted material parameters further reveal that $G_{12}$ indeed differs by $\sim 6 \%$ from $G_{13}$, indicating that the laminate does not possess perfect transversal isotropic symmetry, as expected.

\section{Conclusions}

A two-step optimization procedure, on the basis of a genetic algorithm, has been described in order to inversely extract local material parameters from a P-UPS experiment. First, an analysis on the basis of the Christoffel equation is performed in order to match critical bulk wave angle profiles with the characteristic low-amplitude (or equivalently high curvature)
P-UPS contours. It has been found that this is not sufficient to accurately extract the elasticity tensor $C_{i j}$. The next step of the inversion procedure uses the elasticity tensor $C_{i j}$ obtained in the first step as a starting point to match the characteristic contours of the P-UPS simulation with the contours of the P-UPS recording. To improve the computational performance, the second step has been limited to a few P-UPS sectors, shortening the whole processing time to less than $5 \mathrm{~min}$. The inversion procedure has been demonstrated for synthetic data with and without added noise as well as for P-UPS experiments of annealed aluminum (in different health conditions), cold-rolled DC-06 steel and $\left[0^{\circ}\right]_{8}$ carbon fiber reinforced laminate. Comparison with material parameters obtained from literature on the one hand and from conventional testing on the other hand, yields satisfying agreement.

Acknowledgments Mathias Kersemans acknowledges funding of the FWO-Vlaanderen through grant G012010N.

\section{References}

1. Amenabar M, Mendikute A, López-Arraiza A, Lizaranzu M, Aurrekoetxea J (2011) Comparison and analysis of non-destructive testing techniques suitable for delamination inspection in wind turbine blades. Compos Part B 42:1298-1305

2. Conrad M, Sayir M (2001) Composite ceramic-metal plates tested with flexural waves and holography. Exp Mech 41:412-420

3. Brault R, Germaneau A, Dupre JC, Doumalin P, Mistou S, Fazzini M (2013) In-situ analysis of laminated composite materials by X-ray micro-computed tomography and digital volume correlation. Exp Mech 53:1143-1151

4. Ifju PG, Han B (2010) Recent applications of moire interferometry. Exp Mech 50:1129-1147

5. Buffiere JY, Maire E, Adrien J, Masse JP, Boller E (2010) In situ experiments with $\mathrm{X}$ ray tomography: an attractive tool for experimental mechanics. Exp Mech 50:289-305

6. Maynard J (1996) Resonant ultrasound spectroscopy. Phys Today 49: 26-31

7. Muller M, Sutin A, Guyer R, Talmant M, Laugier P, Johnson PA (2005) Nonlinear resonant ultrasound spectroscopy (NRUS) applied to damage assessment in bone. J Acoust Soc Am 118:3946-3952

8. Ohtani T, Ishii Y (2012) Nonlinear Resonant Ultrasound Spectroscopy (NRUS) applied to fatigue damage evaluation in a pure copper. In: Kamakura T, Sugimoto N (eds) Nonlinear acoustics: state-of-the-art and perspectives. American Institute of Physics, Melville, pp 204-207

9. Solodov I, Bai JX, Busse G (2013) Resonant ultrasound spectroscopy of defects: case study of flat-bottomed holes. J Appl Phys 113: 223512

10. Baudouin S, Hosten B (1996) Immersion ultrasonic method to measure elastic constants and anisotropic attenuation in polymer-matrix and fiber-reinforced composite materials. Ultrasonics 34:379-382

11. Rokhlin SI, Wang W (1992) Double through-transmission bulk wave method for ultrasonic phase-velocity measurement and determination of elastic-constants of composite-materials. J Acoust Soc Am 91: 3303-3312 
12. Balasubramaniam K, Rao NS (1998) Inversion of composite material elastic constants from ultrasonic bulk wave phase velocity data using genetic algorithms. Compos B Eng 29:171-180

13. Wu TT, Liu YH (1999) On the measurement of anisotropic elastic constants of fiber-reinforced composite plate using ultrasonic bulk wave and laser generated Lamb wave. Ultrasonics 37:405-412

14. Vishnuvardhan J, Krishnamurthy CV, Balasubramaniam K (2007) Genetic algorithm reconstruction of orthotropic composite plate elastic constants from a single non-symmetric plane ultrasonic velocity data. Compos B Eng 38:216-227

15. Reddy SSS, Balasubramaniam K, Krishnamurthy CV, Shankar M (2005) Ultrasonic goniometry immersion techniques for the measurement of elastic moduli. Compos Struct 67:3-17

16. Castaings M, Hosten B, Kundu T (2000) Inversion of ultrasonic, plane-wave transmission data in composite plates to infer viscoelastic material properties. NDT E Int 33:377-392

17. Karim MR, Mal AK, Barcohen Y (1990) Inversion of leaky lamb wave data by simplex algorithm. J Acoust Soc Am 88:482-491

18. Cawley P, Hosten B (1997) The use of large ultrasonic transducers to improve transmission coefficient measurements on viscoelastic anisotropic plates. J Acoust Soc Am 101:1373-1379

19. Wang L, Yuan FG (2007) Group velocity and characteristic wave curves of lamb waves in composites: modeling and experiments. Compos Sci Technol 67:1370-1384

20. Vishnuvardhan J, Krishnamurthy CV, Balasubramaniam K (2007) Genetic algorithm based reconstruction of the elastic moduli of orthotropic plates using an ultrasonic guided wave singletransmitter-multiple-receiver SHM array. Smart Mater Struct 16: $1639-1650$

21. Sale M, Rizzo P, Marzani A (2011) Semi-analytical formulation for the guided waves-based reconstruction of elastic moduli. Mech Syst Signal Process 25:2241-2256

22. Qu JM, Berthelot Y, Li ZB (1996) Dispersion of guided circumferential waves in a circular annulus

23. Wilcox PD (1998) Lamb Wave Inspection of Large Structures using Permanently Attached Transducers. Imperial College of Science, Technology and Medicine (London); PhD Thesis 223

24. Towfighi S, Kundu T (2003) Elastic wave propagation in anisotropic spherical curved plates. Int J Solids Struct 40:5495-5510

25. Vandreumel WHM, Speijer JL (1981) Non-destructive composite laminate characterization by means of ultrasonic polar-scan. Mater Eval 39:922-925

26. Degrieck J, Declercq NF, Leroy O (2003) Ultrasonic polar scans as a possible means of non-destructive testing and characterisation of composite plates. Insight 45:196-201

27. Kersemans M, Van Paepegem WM, Van Den Abeele AK, Pyl L, Zastavnik F, Sol H, Degrieck J (2013) The quasi-harmonic ultrasonic polar scan for material characterization: experiment and numerical modeling. Submitted to Ultrasonics

28. Declercq NF, Degrieck J, Leroy O (2006) Simulations of harmonic and pulsed ultrasonic polar scans. NDT E Int 39:205-216

29. Satyanarayan L, Vander Weide JM, Declercq NF (2010) Ultrasonic polar scan imaging of damaged fiber reinforced composites. Mater Eval 68:733-739

30. Kersemans M, De Baere I, Degrieck J, Van Den Abeele K, Pyl L, Zastavnik F, Sol H, Van Paepegem W (2014) Nondestructive damage assessment in fiber reinforced composites with the pulsed ultrasonic polar scan. Polym Test 34:85-96

31. Kersemans M, Van Paepegem W, Van Den Abeele K, Pyl L, Zastavnik F, Sol H, Degrieck J (2014) The pulsed ultrasonic backscatter polar scan and its applications for NDT and material characterization. Exp Mech. doi:10.1007/s11340-013-9843-1

32. Degrieck J (1996) Some possibilities of nondestructive characterisation of composite plates by means of ultrasonic polar scans. In: Van
Hemelrijck D, Anastassopoulos A (eds) Emerging technologies in nondestructive testing (ETNDT). A.A. Balkema, Patras, pp 225-235

33. Declercq NF, Degrieck J, Leroy O (2004) On the influence of fatigue on ultrasonic polar scans of fiber reinforced composites. Ultrasonics 42:173-177

34. Declercq NF, Degrieck J, Leroy O (2006) Ultrasonic polar scans: numerical simulation on generally anisotropic media. Ultrasonics 45 : 32-39

35. Kersemans M, Van Den Abeele K, Lammens N, Degrieck J, Zastavnik F, Gu J, Pyl L, Sol H, Van Paepegem W (2013) Determination of the fiber direction and the C-tensor of a UD carbon/epoxy composite by means of the ultrasonic polar scan. In: Siong GW, Piang LS, Cheong KB (eds) Proceedings of the 2013 International Congress on Ultrasonics (ICU 2013), Singapore

36. Rose JL (1999) Ultrasonic waves in solid media. Cambridge University Press, Cambridge

37. Marzani A, De Marchi L (2013) Characterization of the elastic moduli in composite plates via dispersive guided waves data and genetic algorithms. J Intell Mater Syst Struct 24:2135-2147

38. Wang L, Rokhlin SI (2001) Stable reformulation of transfer matrix method for wave propagation in layered anisotropic media. Ultrasonics 39:413-424

39. Rokhlin SI, Wang L (2002) Ultrasonic waves in layered anisotropic media: characterization of multidirectional composites. Int J Solids Struct 39:5529-5545

40. Wang L, Rokhlin SI (2003) Ultrasonic wave interaction with multidirectional composites: modeling and experiment. J Acoust Soc Am 114:2582-2595

41. Kersemans M, Van Paepegem W, Van Den Abeele K, Pyl L, Zastavnik F, Sol H, Degrieck J (2013) Pitfalls in the experimental recording of ultrasonic polar scans for composite material characterization. Submitted to Ultrasonics

42. Kersemans M, Lammens N, Degrieck J, Van Den Abeele K, Pyl L, Zastavnik F, Sol H, Van Paepegem W (2013), Extraction of bulk wave characteristics from a pulsed ultrasonic polar scan. Submitted to Wave Motion

43. Safaei M (2013) Constitutive modelling of anisotropic sheet metals based on a non-associated flow rule. Ghent University, Faculty of engineering sciences and architecture (Ghent), PhD thesis 246

44. Lebensohn RA, Tome CN (1993) A self-consistent anisotropic approach for the simulation of plastic-deformation and texture development of polycrystals - application to zirconium alloys. Acta Metall Mater 41:2611-2624

45. Sayers CM (1982) Ultrasonic velocities in anisotropic polycrystalline aggregates. J Phys D Appl Phys 15:2157-2167

46. Soden PD, Hinton MJ, Kaddour AS (1998) Lamina properties, layup configurations and loading conditions for a range of fibrereinforced composite laminates. Compos Sci Technol 58:1011-1022

47. Degrieck J (1990) Analyse van impact op een vezelversterkte kunststof (In Dutch). Ghent University, Faculty of Applied Sciences, (Ghent), PhD Thesis

48. Chamis CC (1984) Mechanics of composite materials: past, present and future. In: NASA Technical Memorandum 100793

49. Puck A (1967) Grundlagen der spannungs und veromungs to analyse. Dopl. ing. Kunstoffe, C57

50. Foye RL (1966) An evaluation of various engineering estimates of the transverse properties of unidirectional composites. In: Tenth national SAMPE symposium, San Diego, California, pp 31

51. Daggumati S (2011) Concurrent Modelling and Experimental Analysis of Meso-Scale Strain Fields and Damage in Woven Composites under Static and Fatigue Tensile Loading. Ghent University, Faculty of engineering sciences and architecture (Ghent), $\mathrm{PhD}$ thesis 